\title{
Plasma metabolomic biomarkers of mixed nuts exposure inversely correlate with severity of metabolic syndrome
}

\author{
Ximena Mora-Cubillos ${ }^{1}$, Sara Tulipani ${ }^{1,2}$, Mar Garcia-Aloy ${ }^{1}$, Mònica Bulló ${ }^{3,4}$, \\ Francisco J Tinahones ${ }^{2,4}$, Cristina Andres-Lacueva ${ }^{1} *$
}

\begin{abstract}
${ }^{1}$ Biomarkers \& Nutrimetabolomic Lab., Nutrition and Food Science Department, XaRTA, INSA, Campus Torribera, Pharmacy Faculty, University of Barcelona, Spain

${ }^{2}$ Biomedical Research Institute (IBIMA), Service of Endocrinology and Nutrition, Malaga Hospital Complex (Virgen de la Victoria), Campus de Teatinos s/n, University of Malaga, Malaga, Spain

${ }^{3}$ Human Nutrition Unit, IISPV, Universitat Rovira i Virgili, Reus, Spain

${ }^{4}$ CIBER Fisiopatología de la Obesidad y Nutrición (CIBERobn), Instituto de Salud Carlos III (ISCIII), Madrid, Spain
\end{abstract}

*Correspondence: Dr. Cristina Andres-Lacueva, Biomarkers \& Nutrimetabolomic Lab., Nutrition and Food Science Department, Campus Torribera, Pharmacy Faculty, University of Barcelona, Av. Joan XXIII s/n, 08028 Barcelona, Spain E-mail: candres@ub.edu Fax: +34-93-403-5931

\begin{abstract}
Abbreviations: AUC, area under the curve; CT, control group; CVD, cardiovascular disease; ETs, ellagitannins; HCA, hierarchical cluster analysis; MetS, metabolic syndrome; MUFAs, monounsaturated fatty acids; MVA, multivariate analyses; NU, nuts intake group; OSC, orthogonal signal correction; OSC-PLS-DA, partial least squares discriminant analysis with orthogonal signal correction; PCA, Principal Components Analysis; PIS, Product Ion Scan; PUFAs, polyunsaturated fatty acids; $\mathbf{Q}^{2}$ cum, model predictive ability parameter according to cross validation; ROC, receiver operating curve; $\mathbf{R}^{2} \mathbf{X}$, goodness-of- fit parameter; $\mathbf{R}^{2} \mathbf{Y}$, proportion of the variance of the response variable that is explained by the model; T0, baseline time; T3, after 12-w of nuts consumption; VIP, variable importance for projection; XIC, extracted ion chromatogram.
\end{abstract}

Keywords: adiposity / biomarker of nuts / gut microbiota / metabolomics / plasma human

\section{ABSTRACT}

Scope. To identify the most discriminant dietary biomarkers of nuts exposure in subjects with metabolic syndrome (MetS), and investigate the potential association between exposure and the severity of the MetS diagnostic traits.

Methods and results. We applied the untargeted LC-ESI-qToF-MS-driven metabolomic workflow to explore the changes occurring in the plasma metabolome of MetS subjects following 12-week intake of mixed nuts $(30 \mathrm{~g} / \mathrm{d}$ ) (nuts versus control groups). Urolithin A glucuronide was the most discriminative biomarker of nut exposure, showing the highest predictive capacity [ROC AUC $=89.6 \%$ (80.8-98.4)] despite the inter-individual variation expected for a host-microbial cometabolite. Furthermore, the detection of urolithin A glucuronide in plasma showed significant inverse correlation with basal abdominal adiposity (waist circumference: $r=-0.550$, $p<0.01$; waist-hip ratio: $\mathrm{r}=-0.409, p<0.05)$ and impaired glycemic control (fasting insulin: $\mathrm{r}=-0.414 \&$ HOMA-IR: $\mathrm{r}=-0.417, p<0.05)$. Significant changes in mediumchain dicarboxylic acids, recognized as alternative energy substrates that are particularly 
relevant in the case of glycemic control impairment, were also associated with nut consumption.

Conclusions. Higher levels of urolithin are reported in subjects with less severe MetS traits, especially in females. We believe that this inverse correlation may be related with profile of gut microbial dysbiosis, recently associated to subjects with MetS.

\section{INTRODUCTION}

Tree nuts such as walnuts (Juglans regia L.), almonds (Prunus dulcis (Mill.) D.A. Webb) and hazelnuts (Corylus avellana L.) are key healthy components of several dietary patterns such as the Mediterranean diet [1] and the New Nordic Diet [2], and are also emphasized in the recently updated Dietary Guidelines for Americans [3]. The protective effects of nut consumption have been particularly evidenced against the constellation of interrelated metabolic complications that characterize the metabolic syndrome (MetS) [4]. Both clinical [5] and epidemiological studies [6-8] have associated nut intake with improvements in the diagnostic parameters of MetS, namely abdominal obesity [9], hyperglycemia [3] hypertension [10] and dyslipidemia, and with the reduction of CVD [1, 11-13] through reduction of inflammation and oxidative stress and, enhancement of the endothelial function $[14,15]$.

Despite the huge amount of literature in this regard, the nuts bioactives are not fully characterized, hence the need for hypothesis-generating research to identify unexplored mechanisms of nut exposure and effect. The implication in the observed health effects of minority components besides the well-known macro- and micronutrients contained in nuts (e.g. MUFAs and n-3/n-6 PUFAs, proteins rich in essential amino acids, vitamins, biogenic amines and fiber) $[4,12]$ only attracted attention recently, as well as the role of diet and host-microbial interactions in the health outcomes. The intake of the most abundant polyphenols in walnuts, namely ellagitannins (ETs) [16] has particularly been associated with improvements of metabolic disorders, hence acquiring a growing interest in the food industry as potential functional ingredient [17]. However, due to their high molecular weight, ETs require gut microbial biotransformation to be metabolized into smaller compounds (urolithins) absorbable in the intestine, and the necessary enzymatic machinery does not seem to be ubiquitous among bacterial strains $[18,19]$. In turn, diagnostic components of MetS such as obesity and impaired glycemic control are known to be associated with alterations in the gut microbiota composition (dysbiosis) [20], thus the urolithin-producing capacity may be affected by the presence of these cardiometabolic risk factors, thereby impacting on the bioavailability of bioactives from nut polyphenols.

Nutritional metabolomics is a highly relevant research domain of modern nutrition [21] since, mapping the metabolic fate of food components in biological specimens, it provides more objective measures of food exposure (biomarker discovery) and their effects in the prevention and reduction of disease risk [22]. Due to its data-driven approach, untargeted metabolomics especially offers a valuable tool for identifying new nutritional biomarkers without the need for a starting hypothesis, hence helping to decipher unexplored mechanisms of action and so linking dietary exposure to clinical outcomes [23]. Although the application of nutritional metabolomics has been focused so far to the analysis of the urinary metabolome [29], blood plasma is a particularly informative biosample. Blood in fact gives a snapshot of what is actually transported at the systemic level to and from tissues, even hours following the last intake (permanence in circulation and bioaccessibility to body compartments), thereby sustaining the 
potential bioactivity of the dietary components (association between exogenous and endogenous diet-related changes) [23, 26].

We recently analysed the changes occurring in the urinary metabolome after a 12-week regular consumption of mixed nuts in subjects with diagnosed MetS [16, 27]. To go forward with the hypothesis generated by the previous studies, here we aimed to identify the most discriminant biomarkers of nuts exposure still circulating in $12 \mathrm{~h}$ fasted plasma, and then investigate the potential direct or inverse association between exposure and the severity of the MetS diagnostic traits, at baseline and post-intervention.

\section{EXPERIMENTAL SECTION}

\section{Subjects in Study}

Fifty male and female volunteers aged between 18 and 65 years with at least three MetS risk factors as defined by the Adult Treatment Panel III [28, 29] were recruited in a prospective, randomized, controlled, parallel-designed, 12-week interventional feeding trial (ISRCTN36468613). The design of the trial has been reported in detail previously $[14,15]$. Briefly, participants were stratified by sex and age ( $\leq 50$ or $>50$ years) and then randomly assigned to the control (CT) or the nuts (NU) intervention groups. Both groups received the same qualitative dietary recommendations according to the AHA dietary guidelines [30] in order to follow a low-fat diet. The NU group received a daily supplement of $30 \mathrm{~g}$ of raw mixed nuts with skin ( $15 \mathrm{~g}$ of walnuts, $7.5 \mathrm{~g}$ of almonds and $7.5 \mathrm{~g}$ of hazelnuts), while the CT group was recommended to avoid consumption of nuts. Information about the food intake of the subjects and their adherence to the interventions was collected by 3-day food records at baseline and in control visits, namely every 4 weeks during the trial, and energy, nutrient and polyphenol intakes were calculated as previously described [16]. Good adherence to the dietary recommendations was reported for both intervention groups [14, 15].

Fasting blood samples were collected at baseline (T0) and after 12 weeks of nut consumption (T3) and centrifuged at $2500 \mathrm{rpm}$ for $10 \mathrm{~min}$ at $4{ }^{\circ} \mathrm{C}$. For metabolomic analysis, plasma EDTA aliquots were separated and stored at $-80{ }^{\circ} \mathrm{C}$ until further analysis. Three subjects were excluded from the metabolomic analysis due to evidence of haemolysis in the stored plasma (final sampling: $\mathrm{n}=23 \mathrm{CT}$ group, $\mathrm{n}=24 \mathrm{NU}$ group).

\section{Material and Methods}

\section{Standards and Reagents}

$\alpha$-hydroxyisobutyric acid, gallic acid, L-phenylalanine, L-tryptophan, (-)-epicatechin, syringic acid, sebacic acid, dodecanedioic acid, glycochenodeoxycholic acid, 1-Ostearoyl-sn-glycero-3-phosphocholine, glycocholic acid-(glycyl-1- $\left.{ }^{13} \mathrm{C}\right)$ monohydrate, indole-3-acetic-2,2- $\mathrm{d}_{2}$ acid, acetyl- $\mathrm{d}_{3}-\mathrm{L}$-carnitine hydrochloride and reference human plasma were purchased from Sigma-Aldrich (St. Louis, MO, USA), 4-hydroxyhippuric acid from PhytoLab GmbH \& Co. KG (Germany) and naringenin from Extrasynthèse (Genay, France). Urolithin A glucuronide was chemically synthesized by Kylolab S.A. (Murcia, Spain). UHPLC-MS-grade methanol, acetone, formic acid and HPLC-grade acetonitrile were purchased from Scharlau Chemie S.A. (Barcelona, Spain). Ultrapure water (Milli-Q) was obtained from a Milli-Q Gradient A10 system (Millipore, Bedford, MA). An aqueous standard mix (QC2) composed of exogenous and endogenous compounds of the plasma metabolome chemical variety was prepared (details in Supporting Information Table S1). Aqueous solutions of isotopically labelled and unlabelled compounds were also prepared for use as internal standard (IS $S_{\text {mix }}$ : glycocholic acid-(glycyl-1- ${ }^{13} \mathrm{C}$ ) monohydrate and 1-O-stearoyl-sn-glycero-3- 
phosphocholine, $25 \mathrm{ppm})$ and external standard mixtures $\left(\mathrm{ES}_{\mathrm{mix}}\right.$ : indole-3-acetic-2,2- $\mathrm{d}_{2}$ acid and acetyl- $\mathrm{d}_{3}$-L-carnitine hydrochloride, $25 \mathrm{ppm}$ ), during sample extraction.

\section{Sample Extraction and Data Acquisition}

Plasma samples $(50 \mu \mathrm{L})$ were extracted through in-plate deproteinization by acidic solvent precipitation (acetonitrile, $1 \%$ formic acid) followed by phospholipid SPEmediated removal (Ostro ${ }^{\mathrm{TM}}$, Waters), according to our previously published protocol $[31,32]$. The extracts were analyzed by a LC-ESI-qToF-MS-driven untargeted metabolomic pipeline (Agilent 1200 Series Rapid Resolution HPLC system coupled to a QSTAR Elite System mass spectrometer, Applied Biosystems/MDS Sciex, Framingham, MA, USA.) in negative ionization mode $(70-850 \mathrm{~m} / \mathrm{z})$. Data were acquired using Analyst QS 2.0 software (AB Sciex Toronto, Ontario, Canada). Three types of quality control (QC) were included in the injection plate design to check for the analytical quality grade: QC1, Milli-Q water samples; QC2, aqueous solution of a standard metabolite mix (5 ppm final standard concentration); QC3, randomly selected biological samples reinjected in opposite positions within each batch. Details of analytical protocol in Supporting Information Tables S1 and S2.

\section{Data Analysis and Metabolite Identification}

Mass feature extraction by peak finding and alignment was carried out by using MarkerView $^{\mathrm{TM}}$ 1.2.1 software (AB Sciex, Toronto, Ontario, Canada), as detailed in Supporting Information Table S3. Prior to MVA, the data set was filtered out to remove any ions that did not appear in more than $35 \%$ of the samples of each class separately [33]. Data were then log transformed (to improve normality) and Pareto scaled [34]. The differences in the plasma metabolome due to the 'diet' (NU vs CT) and 'time of collection' factors (T0 vs T3) were explored in the OSC-filtered data set through a partial least squares discriminant analysis (PLS-DA) (SIMCA 13.0 software, Umetrics, Umeå, Sweden). The predictive ability of the PLS models was evaluated through the $R^{2} X, R^{2} Y$, cross validation $\left(Q^{2}\right.$ cum) and permutation test $(n=200$; plot and $\mathrm{CV}$ ANOVA). As a final quality test, the whole data set was randomly split into five equal-size subsamples ( $20 \%$ of samples each), four of which were used as training set whereas the remaining were used as validation set. This process was repeated five times, each subsample being used as the validation set at least once, and the correctly classified individuals in each validation set (\%) were calculated (Supporting Information Table S4). The importance of mass features in the discrimination among classes was visualized by plotting the Variable Importance Projection (VIP) values. To avoid false positives, the significant mass features explaining the class separation were selected by combining the VIP cut-off (VIP $\geq 2$ ), the weight (p) of each metabolite within the statistical model $(|p \geq 0.05|)$ and its correlation with the modelled class designation $(\mid \mathrm{p}$ (corr) $\geq 0.5 \mid)$ as previously described [27], and then submitted to the metabolite identification procedure. First, a two-way HCA based on Pearson correlation and Ward's distance method was performed to determine eventual clusters of mass features originating from the same metabolite (i.e. in-source molecular fragments, adducts, ${ }^{13} \mathrm{C}$ isotopes) and so reduce the queried masses (PermutMatrix 1.9.3 software). Plausible identification hypotheses were then generated (MAIT R package, Rstudio 3.0.1 Inc. $[35,36])$ by matching experimental mass clusters with both in-house and Web-accessible mass spectral databases [37-39] ( $\pm 10 \mathrm{mDa}$ mass error tolerance). Identity confirmation was finally carried out by matching peak chromatographic and MS responses (XIC, PIS) to those of commercial reference standards, when available, spiked in Milli-Q water and plasma (50 ppb final concentration). Finally, the 
performance of the level 1 identified metabolites (Metabolomics Standards Initiative criteria, [40]) in discriminating nut exposure was evaluated by constructing receiver operating characteristic (ROC) curves on the OSC-filtered data set, and estimating the area under the curve [AUC (95\% CI)] values (pRoc R package, Rstudio 3.0.1 Inc.) [41, 42].

\section{Biomarker Performance and Association with MetS Traits}

After checking for data distribution normality, Pearson's correlation coefficients ( $r$ ) were calculated between plasma metabolite levels (peak area) and anthropometric and laboratory measures previously described [14] (SPSS 21.0 software, SPSS Inc., Chicago, IL, USA). Pearson's correlations were performed with dataset at baseline and after the intervention (post-intervention).Variables were grouped into four main categories related cardiometabolic risk (adiposity, glycemic control, lipidemia and blood pressure measures) : a) adiposity markers: waist circumference $(\mathrm{cm})$, body mass index (BMI, calculated as weight in $\mathrm{kg} / \mathrm{height}^{2}$ in $\mathrm{m}^{2}$ ), waist-hip index and body fat (\%); b) glycemic control markers: fasting glucose ( $\mathrm{mmol} / \mathrm{L})$, fasting insulin $(\mu \mathrm{U} / \mathrm{mL})$ and HOMA-IR; c) blood pressure markers: diastolic and systolic blood pressure ( $\mathrm{mm} \mathrm{Hg}$ ); d) blood lipid markers: high-density lipoprotein cholesterol (c-HDL) and triglycerides $(\mathrm{mmol} / \mathrm{L})$. A value of $\mathrm{p}<0.05$ was considered statistically significant.

\section{RESULTS and DISCUSSION}

Data Acquisition Quality. The extraction reproducibility and the LC-MS analytical stability during data acquisition were first confirmed by monitoring the $\mathrm{IS}_{\text {mix }}$ and $\mathrm{ES}_{\text {mix }}$ peak intensity in the plasma samples over time ( $\mathrm{CV}<1 \%$ across the analysis), with no need for data normalization being noticed. As shown in Supporting Information Tables S2 and S3, the run-to-run repeatability of RT and mass accuracies of the QC2 standard metabolite components across the whole data set met the quality criteria proposed for metabolomic analysis protocol (retention time shifts $\leq 0.05 \mathrm{~min}$, mass accuracy deviations $<10 \mathrm{mDa}$ ), and the signal intensity variation was satisfactory (peak areas $\mathrm{CV}<20 \%$ ) except for one metabolite with a very low response to ionization [32, 34]. In addition, PCA analysis was used to display the classification of plasma samples. Details in Supplemental Information Figure S1.

Plasma Metabolomic Biomarkers of Nut Exposure. The OSC filter applied to the processed data set removed 11 components, maintaining the $43 \%$ non-orthogonal variation of the original data set. After confirming the absence of significant metabolomic differences between the two intervention groups at baseline, a three-class model was first built to assess the presence of significant variation within the plasma metabolome at baseline and post intervention (Model 1: T0 vs CT_T3 vs NU_T3 classes). Then, pairwise discriminations between the NU_T3 class and, alternatively, the CT_T3 and T0 classes were carried out and the information resulting from the two models merged, in order to discriminate mass features uniquely up- and downregulated in the NU group, following the intervention (Model 2: T0 vs NU_T3; Model 3: CT_T3 vs NU_T3). The parameters employed to assess the OSC-PLS-DA modelling quality are summarized in Table 1 and in Supporting Information Table S4.

Following the 12-week regular administration of mixed nuts, the plasma metabolome of the Control (CT_T3) and Nuts (NU_T3) groups differed markedly between them and in respect to the baseline samples (Figure 1). A total of twenty-three mass features were responsible for the clear variation in the metabolic fingerprints following nut intake 
(Figure 2). Individual mass features grouped into nineteen ion clusters (including adducts, isotopes, etc..) significantly up- or downregulated following nut exposure (Table 2). Level 1 identification was obtained for three of them, namely a marker of gut microbial-host cometabolism of nut phenolics (urolithin A glucuronide, upregulated) and two medium-chain, even-numbered dicarboxylic acids (dodecanedioic acid and sebacic acid, respectively up- and downregulated). For the latter, the most significant mass feature of the ion cluster corresponded to the dimeric form of the compound $\left([2 \mathrm{M}-\mathrm{H}]^{-}, \mathrm{m} / \mathrm{z}=403.2377\right)$ probably generated in source and co-eluting with the monomer $\left([\mathrm{M}-\mathrm{H}]^{-}, \mathrm{m} / \mathrm{z}=201.1132\right)$. In all cases, the final identification was obtained by overlapping the chromatographic and MS response (XIC, PIS) of the metabolite obtained before and after spiking plasma with the commercially available reference standards (Supporting Information Figure S2). Although several other mass features matched with known theoretical masses (chemical libraries available online,) the low intensity prevented to successfully perform MS/MS experiments, necessary for putative identification (unidentified metabolites).

Biomarkers of Gut Microbial-Host Cometabolism of Nut Polyphenols. Nuts are rich sources of non-ubiquitous classes of polyphenols, namely hydrolyzable tannins (i.e. ETs in walnuts) and condensed tannins (i.e. proanthocyanidins in hazelnuts and almonds), which contribute to the peculiar composition of these fruits and possibly to their health benefits [16, 17]. Walnuts, in particular, are considered a major source of ETs in Mediterranean regions, where nut consumption per person is relatively high compared to other countries [43], in respect to other ET food sources such as berries and pomegranate. These high-molecular-weight polyphenols are known to be subjected to intense gut microbial biotransformation, which is responsible for the production of smaller and more easily absorbed bioactives currently implicated in the protection against cardiometabolic disease risk, namely urolithins (dibenzopyran-6-one derivatives with different hydroxyl substitutions) $[18,19]$.

In the present data-driven study, urolithin A glucuronide was the most discriminant marker of a 12-week intake of nuts detected in the plasma metabolome (VIP > 4, Figure 2). A range of urolithin structure conjugates have been described as the last products of gut microbial-human cometabolism of ETs (i.e. phase II metabolites of urolithin A, B, C and D) and the most stable and detectable forms in different human specimens such as urine, plasma, faeces and tissue [44]. In plasma, conjugated urolithins have been previously described to circulate at concentrations ranging from $\mathrm{nM}$ to low $\mu \mathrm{M}$ (within the sensitivity range of ToF-MS analysis) [45-47], and urolithin A glucuronide was especially detected in plasma of a human volunteer after up to $20 \mathrm{~h}$ from walnut intake [46]. However, the majority of the available data were extrapolated from healthy subjects, with only two reported interventions on MetS individuals [46, 48]. Although different in its analytical nature (non-selective and non-specific LC-MS methodology) and scope (data-driven and hypothesis-generating approach), our plasma metabolomic fingerprinting was in line with previous findings. Overall, our observed findings matched with changes detected in the whole urinary metabolome of the same subjects in the study, as recently described [27], despite the diversity of the (fasted) blood and excretory system physiology. In both biological matrices, in fact, phase II conjugates of urolithin A were the only products of ET metabolism detected through an untargeted approach, with urolithin A glucuronide being the most significant marker among them (Supporting Information Figure S3). Although a subgroup ( 40\%) of the MetS subjects in study revealed to produce other non-A urolithin conjugates by applying targeted LC-MS/MS analysis (e.g. urolithin B metabolites) [16, 44], the 
interindividual variation in the qualitative and quantitative occurrence of these further metabolites hampered them to rise to the role of potential exposure biomarkers, at least when evaluating the study population as a whole, and when applying untargeted metabolomics (non-selective and non-specific analytical nature, data-driven and hypothesis-generating approach).

Biomarkers of Fatty Acid Metabolism. Dodecanedioic acid (C12) and sebacic acid (C10) were respectively up- and down-regulated in fasting plasma following a 12-week exposure to nut intake. The increase in plasma dodecanedioic acid was particularly in line with its increased urinary excretion recently observed [27]. Due to the known occurrence of dicarboxylic acids in some Mediterranean nuts including walnuts, almonds and hazelnuts [27, 49]), dodecanedioic acid may reasonably be a direct biomarker of nuts intake. However, its endogenous origin from the $\omega$ - and $\beta$-oxidative metabolism of MUFAs and PUFAs, highly contained in nuts, cannot be discarded [4, $12,50]$ which could contribute to explain part of the health benefits related the intake of these lipids [3, 51]. In any case, the biological relevance of its increased exposure following nut intake, particularly in subjects with metabolic impairment, may rely on its recognized role as an alternative energy substrate. Once available, in fact, dodecanedioic acid is promptly oxidized and, due to the intermediate biochemical and metabolic characteristics between free fatty acids and glucose, may supply succinylCoA (besides acetyl-CoA, like any other fatty acid) at the end of its $\beta$-oxidation [50], providing an alternative entry into the tricarboxylic acid cycle and a means of metabolic improvement $[52,53]$. The positive effects of dodecanedioic acid infusion have already been described, particularly in those clinical conditions characterized by altered glycemic control, and range from improved mitochondrial function efficiency to reduction of gluconeogenesis, improvement in glycogen synthesis and significant increase in lipid oxidation, in turn leading to reduced body fat mass $[52,54,55]$. The decrease in sebacic acid, in contrast, was not reported previously and may result from an increased downstream or upstream metabolism, suggesting a possible alteration in the metabolism of $\omega$-oxidation of fatty acids in the cytosol of the cells [56, 57]. Finally, no changes in other possible and more expected lipid markers of nut intake, such as $\alpha$ linolenic acid, were observed, in line with previous findings [27], possibly due to the nature of the analytical technique used [58]. Taken together, our data-driven approach would suggest the need to focus attention on 'minor' components of the healthy lipid composition of nuts and their potential health effects, not considered so far, to the best of our knowledge.

Nuts Exposure: Biomarker Performance and Association with Clinical Traits. ROC curves are widely considered to be the most objective and statistically valid method for the evaluation of biomarker performance in metabolomics studies [41]. Both the upregulated metabolites identified in this study showed a good predictive capacity of nut consumption [AUC $=89.6 \%(80.8-98.4)$ and $\mathrm{AUC}=83.7 \%(74.5-$ 92.9), respectively for urolithin A glucuronide and dodecanedioic acid] (Figure 3). Urolithin A glucuronide was the most sensitive and specific plasma biomarker of nut polyphenol intake. These findings are in agreement with recent observations from intervention and cohort studies [27, 59], and suggested how plasma collected at least 12 $\mathrm{h}$ from the last intake may be a valuable alternative to dietary survey and urine metabolic fingerprinting for assessing the consumption of ellagitannin-containing food. The biological significance of urolithins has been extensively investigated in recent research, especially through in vitro experiments, suggesting anti-inflammatory, antiglycative and selective antimicrobial or prebiotic effects [14, 45, 60]. Since they are 
the net result of a gut microbial and host interaction following nut intake, we secondly assessed whether the systemic exposure to urolithin A glucuronide was influenced by the MetS traits known to alter the intestinal microbiota. Table 3 and Supporting Information Figure $\mathbf{S} 4$ show the association between the plasma levels of urolithin A glucuronide following the 12 weeks of nut intake and the severity of the five clinical traits of MetS, at baseline and after the intervention (Pearson correlation coefficient, ${ }^{*} \mathrm{p}$ $<0.05 ; * * p<0.01)$. Correlations ranged from 0.576 (maximum direct correlation) to -0.55 (maximum indirect correlation). A significant inverse correlation was found between the levels of urolithin A glucuronide detected in plasma after nut intake and key parameters of abdominal adiposity (waist circumference: $\mathrm{r}=-0.550, p=0.005$; waist-hip ratio: $-0.409, p=0.047$ ) and impaired glycemic control (fasting insulin: $0.414, p=0.049$; HOMA-IR: $-0.417, p=0.048$ ) at baseline. Linear regression analysis was developed to check for interaction with sex variable. Waist circumference, $p=$ 0.241 ; waist-hip ratio, $p=0.865$; fasting insulin, $p=0.229$; and HOMA-IR, $p=0.185$; It shows that there is no interaction with sex variable. The negative association persisted when testing the correlation with other clinical parameters (e.g. BMI, fasting glucose, percentage of body fat,), although not reaching statistical significance. Furthermore, although the short duration of the dietary intervention allowed the complete remission of the syndrome in only two of the 24 subjects of the NU group (Supporting Information Table S5), the changes in fat percentage following the nut intake positively correlated with levels of urolithin A glucuronide in plasma $(\mathrm{r}=0.456, p=$ 0.025). Similar correlation trends persisted after adjusting for sex and drug treatments. Interestingly, much higher correlation coefficients were observed in female $(n=9)$ than in male $(n=15)$ volunteers, when analyzing the sex response separately (Table 3 ), so that the lack of statistical significance should be interpreted in the context of the reduction of the sample size/statistical power in gender analysis (Supplemental Information Figure S4).

Urolithin A glucuronide did not show similar correlations with the other cardiometabolic risk factors, including hyperlipidemia and hypertension parameters.

These findings allow us to hypothesize that subjects with lower levels of abdominal obesity and insulin resistance may have a microbiota best suited to metabolizing phenolic compounds otherwise indigestible and unabsorbable, such as those derived from nut ETs, once exposed to a dietary challenge, therefore modulating their health impact.

Taken together, the observed associations would confirm the crucial role of the gut microbial community in modulating the production and bioavailability of bioactives from dietary polyphenols. Evidence suggests that the enzymatic machinery responsible for the bioconversion of ETs into urolithins may not be ubiquitously shared across the gut microbial community, but little is still known about the specific strains responsible for urolithin production $[18,19,44]$. Consequently, no data exist to date on the impact that the gut microbial dysbiosis observed in co-occurrence with metabolic alterations may have on the systemic exposure to these bioactives from nut polyphenols. Further research aimed at assessing the prevalence of urolithin-producing bacteria in the gut of metabolically healthy versus diseased individuals is urgently required.

The potential association between the plasma levels of dodecanedioic acid and the severity of the MetS diagnostic traits were also analyzed, at baseline and after the period of nuts intake. Although no significant variations were overall observed, specific gender effects were also noticed (details in Supporting Information Table S6). 


\section{Concluding remarks}

In the present study, the exploration of the plasma metabolome by untargeted metabolomics enabled to identify discriminant metabolites of nut consumption, namely conjugated urolithins and fatty acid metabolites such as dodecanedioic acid, in systemic circulation at least $12 \mathrm{~h}$ following the last intake. Nut consumption was recently associated with an increased urinary excretion of the detected metabolites, in both intervention studies and free-living conditions [16, 27, 59], hence our findings represent an important step in the validation of these compounds as biomarkers of nuts exposure. Moreover, the correlations observed between the identified metabolites and the MetS clinical traits provided a first overview of the link between exposure (referred as the combination of 'intake' and 'host-microbial metabolic capacity') and cardiometabolic risk. Although further studies are highly recommended to verify the generated hypotheses, our findings have confirmed the role of a healthy gut microbial community in modulating the production and bioavailability of healthy bioactives from dietary polyphenols.

\section{Acknowledgments}

This research was supported by: PI13/01172 Project (Plan N de I+D+i 2013-2016) from ISCII-Subdirección General de Evaluación y Fomento de la Investigación, cofounded by Fondo Europeo de Desarrollo Regional (FEDER). PI-0557-2013 Project from Fundación Progreso y Salud, Consejería de Salud y Bienestar Social, Junta de Andalucía. Partially funded by JPI HDHL FOODBALL Project (PCIN-2014-133MINECO-Spain), AGL2005-03605 and ISCIII-CIBEROBN. CA-L has received research funding from the International Nut and Dried Fruit Foundation, Reus, Spain. We also thank the award of 2014SGR1566 from the Generalitat de Catalunya' s Agency AGAUR. ST and XM-C acknowledge the Juan de la Cierva fellowship (MINECO), the AGAUR for the predoctoral FI-DGR 2012 fellowship, respectively.

The authors' contributions to the manuscript were as follows: ST, MB and CA-L designed the research; MB and FJT provided the clinical review of the interventional feeding trial in subjects with metabolic syndrome; XM-C, ST, MG-A and CA-L conducted the research; XM-C, and ST performed the samples analyses; XM-C and ST conducted the statistical analysis; XM-C and ST wrote the paper; ST, MG-A, FJT, MB, and CA-L provided critical revision, and ST and CA-L have the primary responsibility for final content. All authors read and approved the final manuscript. None of the authors had any financial or personal conflicts of interest.

\section{References}

[1] Estruch, R., Ros, E., Salas-Salvado, J., Covas, M. I., et al., Primary prevention of cardiovascular disease with a Mediterranean diet. N. Engl. J. Med. 2013, 368, 12791290.

[2] Mithril, C., Dragsted, L. O., Meyer, C., Tetens, I., et al., Dietary composition and nutrient content of the New Nordic Diet. Public Health Nutr. 2013, 16, 777-785.

[3] U.S. Department of Agriculture, U.S. Department of Health and Human Services, Scientific Report of the 2015 Dietary Guidelines Advisor Committee. Government Printing Office: First Print. http://health.gov/dietaryguidelines/2015-scientific-report/ (accessed Sep 7, 2015). 
[4] Salas-Salvado, J., Guasch-Ferre, M., Bullo, M., Sabate, J., Nuts in the prevention and treatment of metabolic syndrome. Am. J. Clin. Nutr. 2014, 100, 399S-407S.

[5] Blanco Mejia, S., Kendall, C. W., Viguiliouk, E., Augustin, L. S., et al., Effect of tree nuts on metabolic syndrome criteria: a systematic review and meta-analysis of randomised controlled trials. BMJ open 2014, 4, e004660.

[6] O'Neil, C. E., Keast, D. R., Nicklas, T. A., Fulgoni, V. L., 3rd, Nut consumption is associated with decreased health risk factors for cardiovascular disease and metabolic syndrome in U.S. adults: NHANES 1999-2004. J. Am. Coll. Nutr. 2011, 30, 502-510.

[7] Fernandez-Montero, A., Bes-Rastrollo, M., Beunza, J. J., Barrio-Lopez, M. T., et al., Nut consumption and incidence of metabolic syndrome after 6-year follow-up: the SUN (Seguimiento Universidad de Navarra, University of Navarra Follow-up) cohort. Public Health Nutr. 2013, 16, 2064-2072.

[8] Ibarrola-Jurado, N., Bullo, M., Guasch-Ferre, M., Ros, E., et al., Cross-sectional assessment of nut consumption and obesity, metabolic syndrome and other cardiometabolic risk factors: the PREDIMED study. PLOS ONE 2013, 8, e57367.

[9] Jackson, C. L., Hu, F. B., Long-term associations of nut consumption with body weight and obesity. Am. J. Clin. Nutr. 2014, 100, 408S-411S.

[10] Guo, K., Zhou, Z., Jiang, Y., Li, W., Li, Y., Meta-analysis of prospective studies on the effects of nut consumption on hypertension and type 2 diabetes mellitus. $J$. Diabetes 2015, 7, 202-212.

[11] Ros, E., Martinez-Gonzalez, M. A., Estruch, R., Salas-Salvado, J., et al., Mediterranean diet and cardiovascular health: Teachings of the PREDIMED study. $A d v$. Nutr. 2014, 5, 330S-336S.

[12] Ros, E., Health benefits of nut consumption. Nutrients 2010, 2, 652-682.

[13] Luo, C., Zhang, Y., Ding, Y., Shan, Z., et al., Nut consumption and risk of type 2 diabetes, cardiovascular disease, and all-cause mortality: a systematic review and metaanalysis. Am. J. Clin. Nutr. 2014, 100, 256-269.

[14] Casas-Agustench, P., Lopez-Uriarte, P., Bullo, M., Ros, E., et al., Effects of one serving of mixed nuts on serum lipids, insulin resistance and inflammatory markers in patients with the metabolic syndrome. Nutr. Metab. Cardiovasc. Dis. 2011, 21, 126135.

[15] Lopez-Uriarte, P., Nogues, R., Saez, G., Bullo, M., et al., Effect of nut consumption on oxidative stress and the endothelial function in metabolic syndrome. Clin. Nutr. 2010, 29, 373-380.

[16] Tulipani, S., Urpi-Sarda, M., Garcia-Villalba, R., Rabassa, M., et al., Urolithins are the main urinary microbial-derived phenolic metabolites discriminating a moderate consumption of nuts in free-living subjects with diagnosed metabolic syndrome. $J$. Agric. Food Chem. 2012, 60, 8930-8940.

[17] Garcia-Munoz, C., Vaillant, F., Metabolic fate of ellagitannins: implications for health, and research perspectives for innovative functional foods. Crit. Rev. Food Sci. Nutr. 2014, 54, 1584-1598.

[18] Selma, M. V., Beltran, D., Garcia-Villalba, R., Espin, J. C., Tomas-Barberan, F. A., Description of urolithin production capacity from ellagic acid of two human intestinal Gordonibacter species. Food Funct. 2014, 5, 1779-1784.

[19] Selma, M. V., Tomas-Barberan, F. A., Beltran, D., Garcia-Villalba, R., Espin, J. C., Gordonibacter urolithinfaciens sp. nov., an urolithin-producing bacterium isolated from human gut. Int. J. Syst. Evol. Microbiol. 2014, 64, 2346-2352.

[20] Hartstra, A. V., Bouter, K. E., Backhed, F., Nieuwdorp, M., Insights into the role of the microbiome in obesity and type 2 diabetes. Diabetes Care 2015, 38, 159-165. 
[21] Scalbert, A., Brennan, L., Manach, C., Andres-Lacueva, C., et al., The food metabolome: a window over dietary exposure. Am. J. Clin. Nutr. 2014, 99, 1286-1308.

[22] Ussar, S., Griffin, N. W., Bezy, O., Fujisaka, S., et al., Interactions between Gut Microbiota, Host Genetics and Diet Modulate the Predisposition to Obesity and Metabolic Syndrome. Cell Metab. 2015, 22, 516-530.

[23] van Ravenzwaay, B., Cunha, G. C., Leibold, E., Looser, R., et al., The use of metabolomics for the discovery of new biomarkers of effect. Toxicol. Lett. 2007, 172, 21-28.

[24] Llorach, R., Garcia-Aloy, M., Tulipani, S., Vazquez-Fresno, R., Andres-Lacueva, C., Nutrimetabolomic strategies to develop new biomarkers of intake and health effects. J. Agric. Food Chem. 2012, 60, 8797-8808.

[25] Nicholson, J. K., Holmes, E., Kinross, J. M., Darzi, A. W., et al., Metabolic phenotyping in clinical and surgical environments. Nature 2012, 491, 384-392.

[26] Rappaport, S. M., Barupal, D. K., Wishart, D., Vineis, P., Scalbert, A., The blood exposome and its role in discovering causes of disease. Environ. Health Perspect. 2014, $122,769-774$.

[27] Tulipani, S., Llorach, R., Jauregui, O., Lopez-Uriarte, P., et al., Metabolomics unveils urinary changes in subjects with metabolic syndrome following 12-week nut consumption. J. Proteome Res. 2011, 10, 5047-5058.

[28] Grundy, S. M., Cleeman, J. I., Daniels, S. R., Donato, K. A., et al., Diagnosis and management of the metabolic syndrome: an American Heart Association/National Heart, Lung, and Blood Institute Scientific Statement. Circulation 2005, 112, 27352752.

[29] Alberti, K. G., Eckel, R. H., Grundy, S. M., Zimmet, P. Z., et al., Harmonizing the metabolic syndrome: a joint interim statement of the International Diabetes Federation Task Force on Epidemiology and Prevention; National Heart, Lung, and Blood Institute; American Heart Association; World Heart Federation; International Atherosclerosis Society; and International Association for the Study of Obesity. Circulation 2009, 120, $1640-1645$.

[30] Krauss, R. M., Eckel, R. H., Howard, B., Appel, L. J., et al., AHA Dietary Guidelines: revision 2000: A statement for healthcare professionals from the Nutrition Committee of the American Heart Association. Circulation 2000, 102, 2284-2299.

[31] Tulipani, S., Llorach, R., Urpi-Sarda, M., Andres-Lacueva, C., Comparative analysis of sample preparation methods to handle the complexity of the blood fluid metabolome: when less is more. Anal. Chem. 2013, 85, 341-348.

[32] Tulipani, S., Mora-Cubillos, X., Jauregui, O., Llorach, R., et al., New and Vintage Solutions to Enhance the Plasma Metabolome Coverage by LC-ESI-MS Untargeted Metabolomics. The Not-So-Simple Process of Method Performance Evaluation. Anal. Chem. 2015, 87, 2639-2647.

[33] Rothwell, J. A., Fillatre, Y., Martin, J. F., Lyan, B., et al., New biomarkers of coffee consumption identified by the non-targeted metabolomic profiling of cohort study subjects. PLoS ONE 2014, 9, e93474.

[34] Llorach, R., Garrido, I., Monagas, M., Urpi-Sarda, M., et al., Metabolomics study of human urinary metabolome modifications after intake of almond (Prunus dulcis (Mill.) D.A. Webb) skin polyphenols. J. Proteome Res. 2010, 9, 5859-5867.

[35] Fernandez-Albert, F., Llorach, R., Andres-Lacueva, C., Perera, A., An R package to analyse LC/MS metabolomic data: MAIT (Metabolite Automatic Identification Toolkit). Bioinformatics 2014, 30, 1937-1939. 
[36] Fernandez-Albert, F., Llorach, R., Andres-Lacueva, C., Perera-Lluna, A., Peak aggregation as an innovative strategy for improving the predictive power of LC-MS metabolomic profiles. Anal. Chem. 2014, 86, 2320-2325.

[37] Wishart, D. S., Jewison, T., Guo, A. C., Wilson, M., et al., HMDB 3.0--The Human Metabolome Database in 2013. Nucleic Acids Res. 2013, 41, D801-807.

[38] Wagele, B., Witting, M., Schmitt-Kopplin, P., Suhre, K., MassTRIX reloaded: combined analysis and visualization of transcriptome and metabolome data. PLOS ONE 2012, 7, e39860.

[39] Horai, H., Arita, M., Kanaya, S., Nihei, Y., et al., MassBank: a public repository for sharing mass spectral data for life sciences. J. Mass Spectrom. 2010, 45, 703-714.

[40] Sumner, L. W., Amberg, A., Barrett, D., Beale, M. H., et al., Proposed minimum reporting standards for chemical analysis Chemical Analysis Working Group (CAWG) Metabolomics Standards Initiative (MSI). Metabolomics 2007, 3, 211-221.

[41] Xia, J., Broadhurst, D. I., Wilson, M., Wishart, D. S., Translational biomarker discovery in clinical metabolomics: an introductory tutorial. Metabolomics 2013, 9, 280-299.

[42] Robin, X., Turck, N., Hainard, A., Tiberti, N., et al., pROC: an open-source package for $\mathrm{R}$ and $\mathrm{S}+$ to analyze and compare ROC curves. BMC Bioinformatics 2011, $12,77$.

[43] Jenab, M., Sabate, J., Slimani, N., Ferrari, P., et al., Consumption and portion sizes of tree nuts, peanuts and seeds in the European Prospective Investigation into Cancer and Nutrition (EPIC) cohorts from 10 European countries. Br. J. Nutr. 2006, 96 Suppl 2, S12-23.

[44] Tomas-Barberan, F., Garcia-Villalba, R., Gonzalez-Sarrias, A., Selma, M. V., Espin, J. C., Ellagic acid metabolism by human gut microbiota: Consistent observation of three urolithin phenotypes in intervention trials, independent of food source, age and health status. J. Agric. Food Chem. 2014, 62, 6535-6538.

[45] Espin, J. C., Larrosa, M., Garcia-Conesa, M. T., Tomas-Barberan, F., Biological significance of urolithins, the gut microbial ellagic Acid-derived metabolites: the evidence so far. Evid. Based Complement. Alternat. Med. 2013, 2013, 270418.

[46] Pfundstein, B., Haubner, R., Wurtele, G., Gehres, N., et al., Pilot Walnut Intervention Study of Urolithin Bioavailability in Human Volunteers. J. Agric. Food Chem. 2014, 62, 10264-10273.

[47] Seeram, N. P., Henning, S. M., Zhang, Y., Suchard, M., et al., Pomegranate juice ellagitannin metabolites are present in human plasma and some persist in urine for up to 48 hours. J. Nutr. 2006, 136, 2481-2485.

[48] Puupponen-Pimia, R., Seppanen-Laakso, T., Kankainen, M., Maukonen, J., et al., Effects of ellagitannin-rich berries on blood lipids, gut microbiota, and urolithin production in human subjects with symptoms of metabolic syndrome. Mol. Nutr. Food Res. 2013, 57, 2258-2263.

[49] Dembitsky, V. M., Goldshlag, P., Srebnik, M., Occurrence of dicarboxylic (dioic) acids in some Mediterranean nuts. Food Chem. 2002, 76, 469-473.

[50] Mingrone, G., Castagneto, M., Medium-chain, even-numbered dicarboxylic acids as novel energy substrates: an update. Nutr. Rev. 2006, 64, 449-456.

[51] Eckel, R. H., Jakicic, J. M., Ard, J. D., de Jesus, J. M., et al., 2013 AHA/ACC guideline on lifestyle management to reduce cardiovascular risk: a report of the American College of Cardiology/American Heart Association Task Force on Practice Guidelines. J. Am. Coll. Cardiol. 2014, 63, 2960-2984.

[52] Mingrone, G., Castagneto-Gissey, L., Mace, K., Use of dicarboxylic acids in type 2 diabetes. Br. J. Clin. Pharmacol. 2013, 75, 671-676. 
[53] Storlien, L., Oakes, N. D., Kelley, D. E., Metabolic flexibility. Proc. Nutr. Soc. 2004, 63, 363-368.

[54] Greco Md, A. V., Mingrone Md, P. G., Capristo Md, E., Benedetti Md, G., et al., The Metabolic Effect of Dodecanedioic Acid Infusion in Non-Insulin-Dependent Diabetic Patients. Nutrition 1998, 14, 351-357.

[55] Salinari, S., Bertuzzi, A., Gandolfi, A., Greco, A. V., et al., Dodecanedioic acid overcomes metabolic inflexibility in type 2 diabetic subjects. Am. J. Physiol. Endocrinol. Metab. 2006, 291, E1021-E1058.

[56] Wang, M., Yang, X., Ren, L., Li, S., et al., Biomarkers identified by urinary metabonomics for noninvasive diagnosis of nutritional rickets. J. Proteome Res. 2014, $13,4131-4142$.

[57] Kolvraa, S., Gregersen, N., In vitro studies on the oxidation of medium-chain dicarboxylic acids in rat liver. Biochim. Biophys. Acta 1986, 876, 515-525.

[58] Nieman, D. C., Shanely, R. A., Luo, B., Meaney, M. P., et al., Metabolomics approach to assessing plasma 13- and 9-hydroxy-octadecadienoic acid and linoleic acid metabolite responses to 75-km cycling. Am. J. Physiol. Regul. Integr. Comp. Physiol. 2014, 307, R68-74.

[59] Garcia-Aloy, M., Llorach, R., Urpi-Sarda, M., Tulipani, S., et al., Novel multimetabolite prediction of walnut consumption by a urinary biomarker model in a free-living population: the PREDIMED study. J. Proteome Res. 2014, 13, 3476-3483.

[60] Garcia-Villalba, R., Beltran, D., Espin, J. C., Selma, M. V., Tomas-Barberan, F. A., Time course production of urolithins from ellagic acid by human gut microbiota. $J$. Agric. Food Chem. 2013, 61, 8797-8806.

\section{Figures}

Figure 1. OSC-PLS-DA score plot (Model 1). Figures and colors indicate the type of plasma samples to improve visualization: baseline samples (CT_T0 and NU_T0) are gray and black circles, CT_T3 are triangles and CT_T3 are pentagons, respectively.

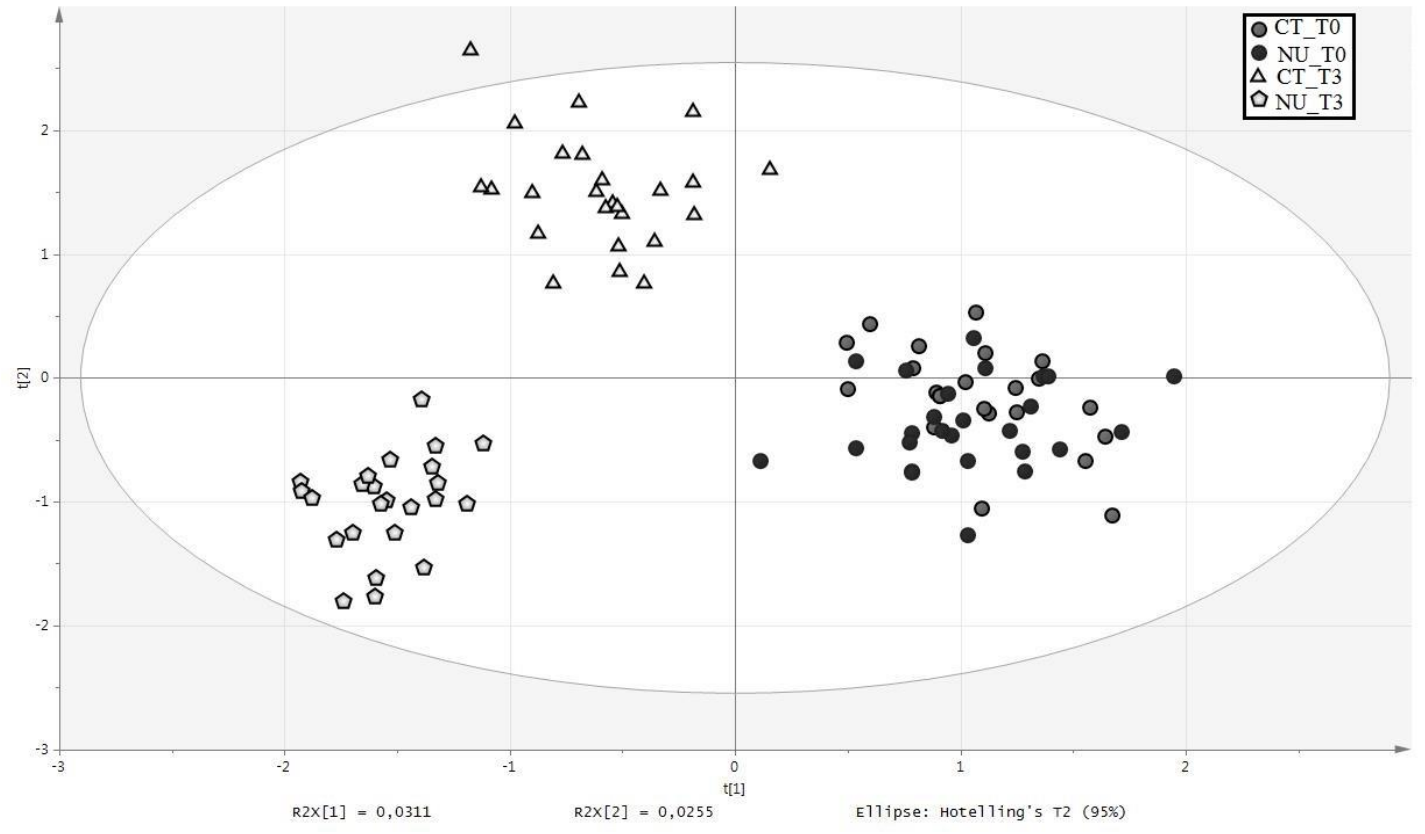


Figure 2. VIP value of models T0 vs NU_T3 and CT_T3 vs NU_T3. Dotted line represent discriminants biomarkers with VIP value $\geq 2$ in both models. Urolithin A glucuronide (black box), dodecanedioic acid (black pentagon) and sebacic acid (monomer and dimer, black diamante) are examples of some discriminant biomarkers.

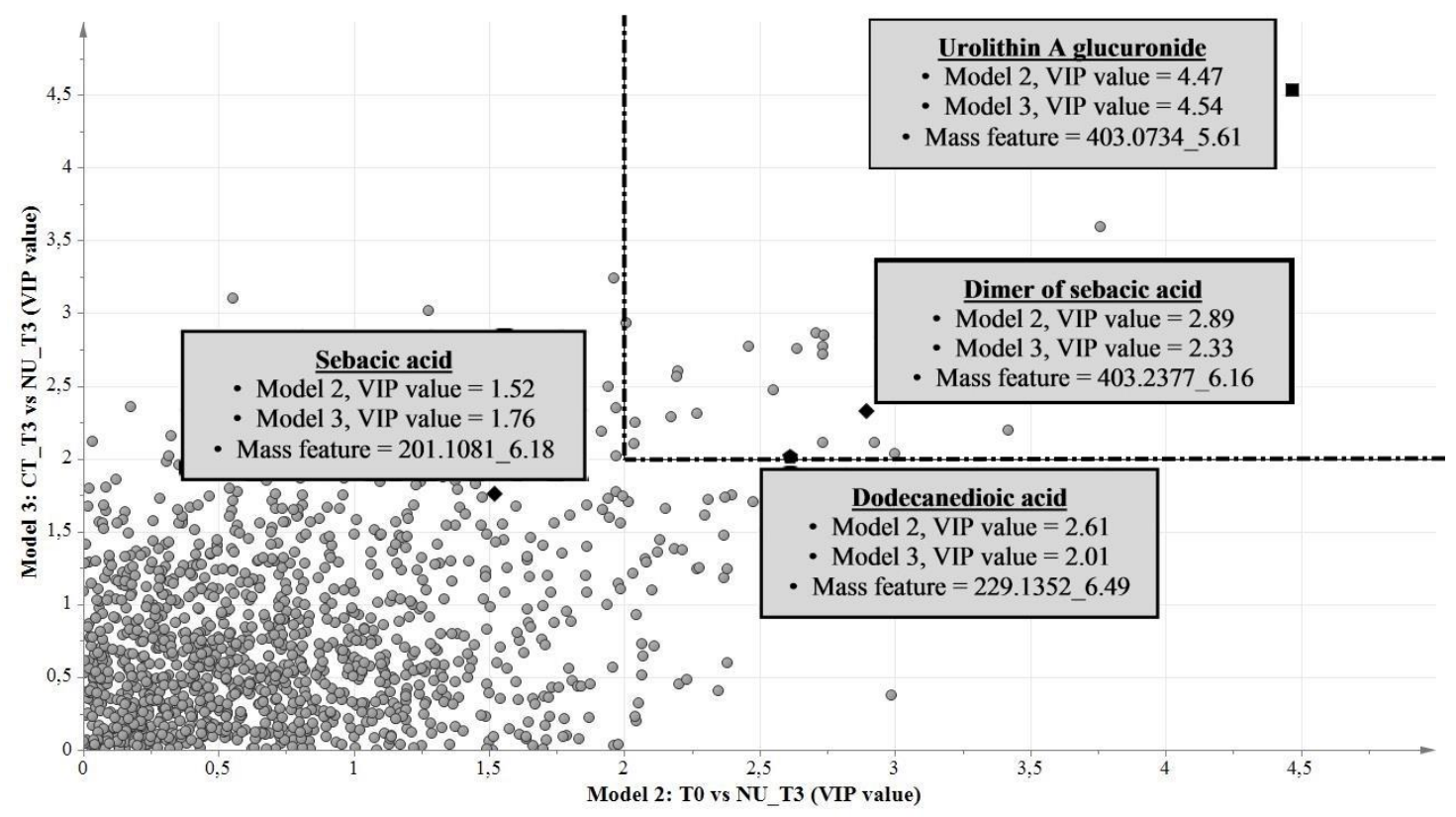


Figure 3. ROC curve analysis of urolithin A glucuronide and dodecanedioic acid (upregulated biomarkers of nuts exposure (NU_T3 subjects vs T0 and CT_T3).

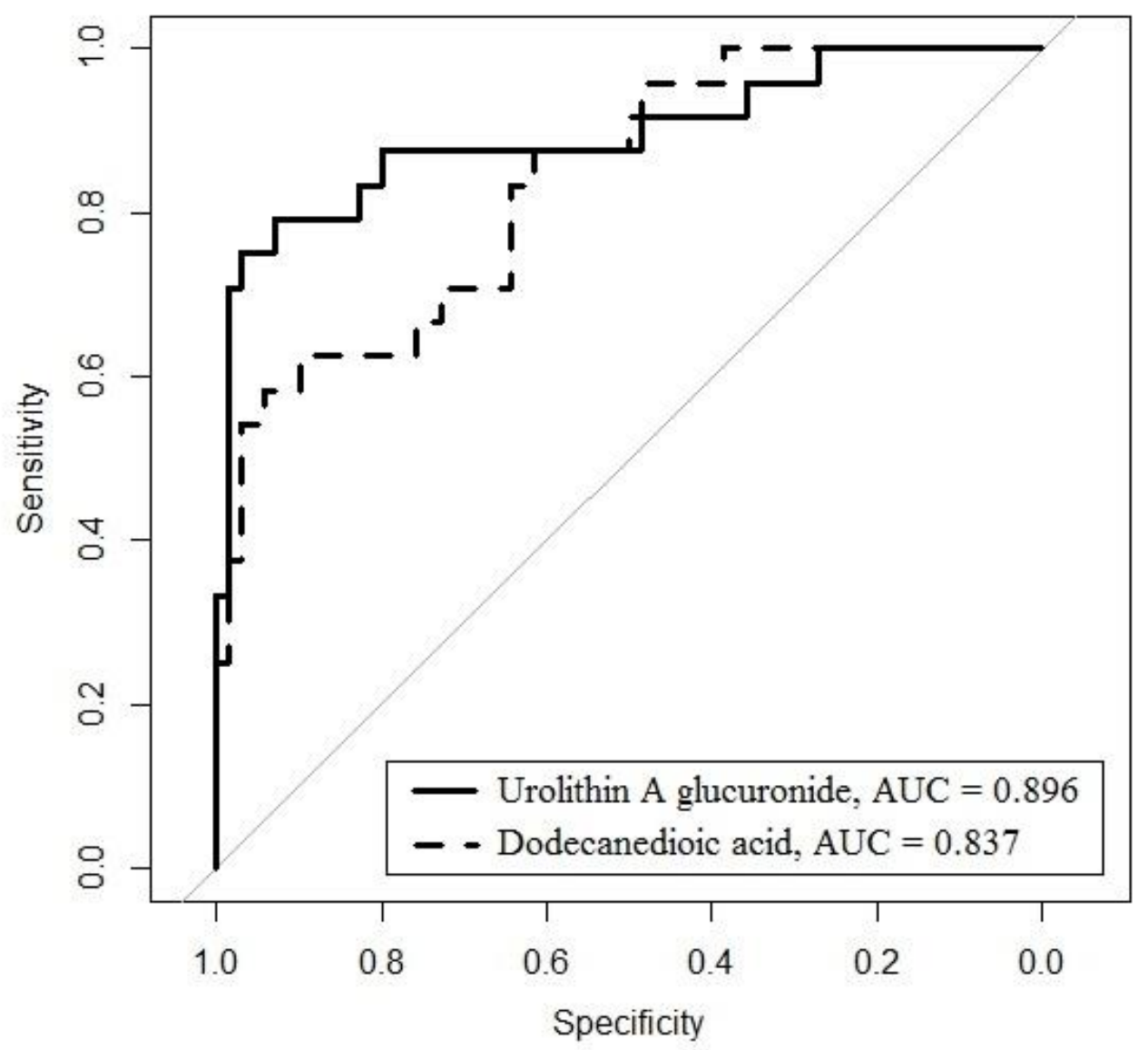




\section{Tables}

Table 1. Summary of parameters for assessing the OSC-PLS-DA modeling quality.

\begin{tabular}{clccccccc}
\hline Model $^{\mathbf{a}}$ & \multicolumn{1}{c}{ No. Sample Classes } & No. $^{\mathbf{b}}$ & $\mathbf{R}^{\mathbf{2}} \mathbf{X}(\mathbf{c u m})^{\mathbf{c}}$ & $\mathbf{R}^{\mathbf{2}} \mathbf{Y}(\mathbf{c u m})^{\mathbf{c}}$ & $\mathbf{Q}^{\mathbf{2}}(\mathbf{c u m})^{\mathbf{c}}$ & $\begin{array}{c}\mathbf{R} \\
\text { intercept }^{\mathbf{d}}\end{array}$ & $\mathbf{Q}_{\text {intercept }^{\mathbf{d}}}$ & $\boldsymbol{p}^{\mathbf{e}}$ \\
\hline 1 & 3 (T0 vs CT_T3 vs NU_T3) & 2 & 0.057 & 0.886 & 0.669 & 0.562 & -0.223 & $8.92 \mathrm{E}-35$ \\
2 & 2 (T0 vs NU_T3) & 1 & 0.040 & 0.942 & 0.744 & 0.645 & -0.147 & $1.83 \mathrm{E}-21$ \\
3 & 2 (CTT3 vs NU_T3) & 1 & 0.047 & 0.928 & 0.621 & 0.709 & -0.152 & $2.07 \mathrm{E}-10$ \\
\hline
\end{tabular}

${ }^{a}$ In model, the generation of the OSC filters removed respectively 11 and 8 components (Eigenvalue > 2), maintaining 52\% and $43 \%$ nonorthogonal variation in the original data set. ${ }^{b}$ Number of components selected. ${ }^{c} R^{2} X(c u m)$ and $R^{2} Y(c u m)$ are the cumulative modeled variation in $\mathrm{X}$ and $\mathrm{Y}$ matrix, respectively, and $\mathrm{Q}^{2} \mathrm{Y}(\mathrm{cum})$ is the cumulative predicted variation in $\mathrm{Y}$ matrix. ${ }^{\mathrm{d}}$ Obtained after a permutation test $(\mathrm{n}=200)$. ${ }^{\mathrm{e}}$ Value from ANOVA based on the cross-validated predictive residuals. The models were considered significant value with $p<0.001$.

Table 2. Identified and nonidentified metabolites associated with nuts intake in the plasma metabolome of MetS subjects ${ }^{\mathrm{a}}$.

\begin{tabular}{|c|c|c|c|c|c|c|c|c|c|c|}
\hline \multirow{2}{*}{$\begin{array}{l}\text { No. } \\
\text { cluster }\end{array}$} & \multirow{2}{*}{ RT (min) } & \multirow{2}{*}{$\begin{array}{l}\text { Detected mass } \\
\qquad(\mathrm{m} / \mathrm{z})^{b}\end{array}$} & \multirow{2}{*}{$\begin{array}{l}\text { Theoretical } \\
\text { mass }(m / z)\end{array}$} & \multirow{2}{*}{$\begin{array}{l}\text { Error } \\
(\mathbf{m D a})\end{array}$} & \multirow{2}{*}{ Assignation } & \multirow{2}{*}{$\begin{array}{c}\text { Potential } \\
\text { biomarker }\end{array}$} & \multirow{2}{*}{ Change $^{c}$} & \multicolumn{2}{|c|}{ VIP value ${ }^{d}$} & \multirow{2}{*}{$\begin{array}{l}\text { Level of } \\
\text { evidence }^{e}\end{array}$} \\
\hline & & & & & & & & $\begin{array}{c}\text { T0 vr } \\
\text { NU_T3 }\end{array}$ & $\begin{array}{c}\text { CT_T3 vr } \\
\text { NU_T3 }\end{array}$ & \\
\hline \multicolumn{11}{|c|}{ Markers of microbial-derived and phase II metabolism of nuts phenolics } \\
\hline 1 & 5.61 & 403.0734 & 403.0671 & -6.35 & {$[\mathrm{M}-\mathrm{H}]^{-}$} & $\begin{array}{l}\text { Urolithin A } \\
\text { glucuronide }\end{array}$ & $\uparrow$ & 4.47 & 4.54 & 1 \\
\hline \multicolumn{11}{|c|}{ Markers of fatty acids metabolism (Medium-chain, even-numbered dicarboxylic acids) } \\
\hline \multirow[t]{2}{*}{2} & 6.16 & 403.2377 & 403.2337 & -4.00 & {$[2 \mathrm{M}-\mathrm{H}]^{-}$} & Sebacic acid & $\downarrow$ & 2.89 & 2.33 & 1 \\
\hline & 6.18 & 201.1081 & 201.1132 & 5.10 & {$[\mathrm{M}-\mathrm{H}]^{-}$} & & $\downarrow$ & 1.52 & 1.76 & \\
\hline
\end{tabular}




\begin{tabular}{|c|c|c|c|c|c|c|c|c|c|}
\hline 3 & 6.49 & 229.1352 & 229.1445 & {$[\mathrm{M}-\mathrm{H}]^{-}$} & $\begin{array}{c}\text { Dodecanedioic } \\
\text { acid } \\
\end{array}$ & $\uparrow$ & 2.61 & 2.01 & 1 \\
\hline \multicolumn{9}{|c|}{ Unidentified markers } & \\
\hline 4 & 0.5 & 352.0023 & & {$[\mathrm{M}-\mathrm{H}]^{-}$} & & $\downarrow$ & 3.76 & 3.59 & \\
\hline 5 & 5.69 & 145.0519 & & {$[\mathrm{M}-\mathrm{H}]^{-}$} & & $\uparrow$ & 3.42 & 2.20 & \\
\hline 6 & 0.60 & 583.1809 & & {$[\mathrm{M}-\mathrm{H}]^{-}$} & & $\uparrow$ & 3.03 & 2.44 & \\
\hline 7 & 0.47 & 605.1648 & & {$[\mathrm{M}-\mathrm{H}]^{-}$} & & $\uparrow$ & 3.00 & 2.04 & \\
\hline 8 & 7.86 & 253.2187 & & {$[\mathrm{M}-\mathrm{H}]^{-}$} & & $\uparrow$ & 2.92 & 2.12 & \\
\hline 9 & 6.63 & 194.0835 & & {$[\mathrm{M}-\mathrm{H}]^{-}$} & & $\downarrow$ & 2.73 & 2.11 & \\
\hline 10 & 6.23 & 527.2204 & & {$[\mathrm{M}-\mathrm{H}]^{-}$} & & $\downarrow$ & 2.73 & 2.72 & \\
\hline \multirow[t]{4}{*}{11} & 6.41 & 421.1493 & & {$[\mathrm{M}-\mathrm{H}]^{-}$} & & $\downarrow$ & 2.71 & 2.86 & \\
\hline & 6.41 & 422.1508 & & $\left.{ }^{13} \mathrm{C}[\mathrm{M}-\mathrm{H}]\right]^{-}$ & & $\downarrow$ & 2.64 & 2.76 & \\
\hline & 6.41 & 423.1468 & & $2^{13} \mathrm{C}[\mathrm{M}-\mathrm{H}]^{-}$ & & $\downarrow$ & 2.73 & 2.77 & \\
\hline & 6.43 & 424.1482 & & $3^{13} \mathrm{C}[\mathrm{M}-\mathrm{H}]^{-}$ & & $\downarrow$ & 2.74 & 2.85 & \\
\hline \multirow[t]{2}{*}{12} & 6.41 & 807.3249 & & {$[\mathrm{M}-\mathrm{H}]^{-}$} & & $\downarrow$ & 2.55 & 2.48 & \\
\hline & 6.41 & 808.3294 & & ${ }^{13} \mathrm{C}[\mathrm{M}-\mathrm{H}]^{-}$ & & $\downarrow$ & 2.17 & 2.29 & \\
\hline 13 & 0.6 & 354.9976 & & {$[\mathrm{M}-\mathrm{H}]^{-}$} & & $\uparrow$ & 2.45 & 2.77 & \\
\hline 14 & 0.6 & 347.0152 & & $\left.{ }^{13} \mathrm{C}[\mathrm{M}-\mathrm{H}]\right]^{-}$ & & $\uparrow$ & 2.27 & 2.31 & \\
\hline 15 & 4.47 & 217.0284 & & {$[\mathrm{M}-\mathrm{H}]^{-}$} & & $\uparrow$ & 2.20 & 2.60 & \\
\hline 16 & 6.38 & 607.2990 & & {$[\mathrm{M}-\mathrm{H}]^{-}$} & & $\downarrow$ & 2.19 & 2.56 & \\
\hline 17 & 6.43 & 475.2045 & & {$[\mathrm{M}-\mathrm{H}]^{-}$} & & $\downarrow$ & 2.04 & 2.25 & \\
\hline \multirow[t]{2}{*}{18} & 5.83 & 317.1167 & & {$[\mathrm{M}-\mathrm{H}]^{-}$} & & $\uparrow$ & 1.99 & 1.74 & \\
\hline & 5.83 & 318.1196 & & ${ }^{13} \mathrm{C}[\mathrm{M}-\mathrm{H}]^{-}$ & & $\uparrow$ & 2.04 & 2.11 & \\
\hline 19 & 5.78 & 175.6309 & & {$[\mathrm{M}-\mathrm{H}]^{-}$} & & $\uparrow$ & 2.01 & 2.94 & \\
\hline
\end{tabular}

${ }^{\text {a }}$ Clusters are listed according the decreasing VIP value; all the proposed mass features met the significance criteria proposed $(|\mathrm{p} \geq 0.05|$ and $\mid \mathrm{p}($ corr $) \geq 0.5 \mid) .{ }^{\mathrm{b}}$ Data obtained by LC-ESI-q-TOF mass spectrometry (see text for details). ${ }^{\mathrm{c}}$ Direction of changes observed following the nuts consumption. ${ }^{\mathrm{d}}$ Calculated from the two-class OSC-PLS-DA models M2 and M3. ${ }^{\mathrm{e}}$ According to Metabolomics Standards iniciative. 
Table 3. Pearson rank correlation coefficient for correlation of changes in plasma levels of urolithin A glucuronide and the severity of the five clinical traits of MetS (waist circumference, fasting glucose, c-HDL, triglycerides and elevated blood pressure-SPB $\&$ DPB) at baseline and after nuts intervention. $* p<0.05 ; * *<0.01$.

\begin{tabular}{|c|c|c|c|c|c|c|}
\hline & \multirow{2}{*}{ Variable } & & \multicolumn{2}{|c|}{ All subjects } & \multirow{2}{*}{$\begin{array}{c}\text { Female } \\
(n=9)\end{array}$} & \multirow{2}{*}{$\begin{array}{c}\text { Male } \\
(n=15)\end{array}$} \\
\hline & & & $\mathbf{r}$ & $\begin{array}{c}\mathbf{r} \\
\text { (adjusted) }\end{array}$ & & \\
\hline \multirow{8}{*}{ 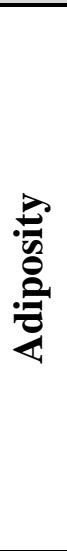 } & Waist circumference $(\mathrm{cm})$ & Baseline & $-0.550 * *$ & $-0.359^{a}$ & -0.517 & -0.272 \\
\hline & & $\begin{array}{l}\text { Post- } \\
\text { intervention }\end{array}$ & 0.184 & $0.128^{\mathrm{a}}$ & 0.406 & -0.056 \\
\hline & Waist-hip ratio & Baseline & $-0.409 *$ & $0.041^{\mathrm{a}}$ & -0.033 & 0.137 \\
\hline & & $\begin{array}{l}\text { Post- } \\
\text { intervention }\end{array}$ & 0.147 & $0.048^{\mathrm{a}}$ & 0.266 & -0.045 \\
\hline & $\mathrm{BMI}\left(\mathrm{kg} / \mathrm{m}^{2}\right)$ & Baseline & -0.228 & $-0.383^{\mathrm{a}}$ & -0.376 & -0.400 \\
\hline & & $\begin{array}{l}\text { Post- } \\
\text { intervention }\end{array}$ & 0.213 & $0.243^{\mathrm{a}}$ & 0.482 & -0.018 \\
\hline & Body fat (\%) & Baseline & 0.222 & $-0.369^{\mathrm{a}}$ & -0.309 & -0.445 \\
\hline & & $\begin{array}{l}\text { Post- } \\
\text { intervention }\end{array}$ & $0.456^{*}$ & $0.309^{\mathrm{a}}$ & 0.576 & 0.224 \\
\hline \multirow{6}{*}{ 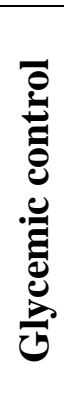 } & Fasting glucose (mmol/L) & Baseline & -0.254 & $-0.202^{\mathrm{a}}$ & -0.272 & -0.184 \\
\hline & & $\begin{array}{l}\text { Post- } \\
\text { intervention }\end{array}$ & 0.272 & $0.322^{\mathrm{a}}$ & 0.396 & 0.333 \\
\hline & Insulin $(\mu \mathrm{U} / \mathrm{mL})$ & Baseline & $-0.414^{*}$ & $-0.277^{\mathrm{a}}$ & -0.501 & -0.128 \\
\hline & & $\begin{array}{l}\text { Post- } \\
\text { intervention }\end{array}$ & 0.205 & $0.084^{\mathrm{a}}$ & 0.2 & -0.021 \\
\hline & HOMA-IR & Baseline & $-0.417 *$ & $-0.265^{\mathrm{a}}$ & -0.522 & -0.115 \\
\hline & & $\begin{array}{l}\text { Post- } \\
\text { intervention }\end{array}$ & 0.226 & $0.094^{\mathrm{a}}$ & 0.199 & 0.013 \\
\hline \multirow{4}{*}{ } & c-HDL (mmol/L) & Baseline & 0.366 & $0.181^{b}$ & 0.402 & 0.103 \\
\hline & & $\begin{array}{l}\text { Post- } \\
\text { intervention }\end{array}$ & -0.312 & $-0.208^{\mathrm{b}}$ & -0.298 & -0.011 \\
\hline & Triglycerides (mmol/L) & Baseline & -0.188 & $-0.090^{\mathrm{b}}$ & -0.399 & 0.024 \\
\hline & & $\begin{array}{l}\text { Post- } \\
\text { intervention }\end{array}$ & 0.108 & $0.143^{\mathrm{b}}$ & 0.265 & -0.115 \\
\hline \multirow{4}{*}{ 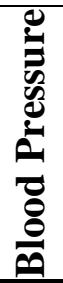 } & SPB (mm Hg) & Baseline & 0.126 & $0.184^{b}$ & 0.321 & -0.065 \\
\hline & & $\begin{array}{l}\text { Post- } \\
\text { intervention }\end{array}$ & -0.062 & $0.186^{\mathrm{b}}$ & 0.144 & 0.246 \\
\hline & $\mathrm{DPB}(\mathrm{mm} \mathrm{Hg})$ & Baseline & 0.242 & $0.304^{\mathrm{b}}$ & 0.181 & 0.432 \\
\hline & & $\begin{array}{l}\text { Post- } \\
\text { intervention }\end{array}$ & 0.092 & $0.032^{\mathrm{b}}$ & 0.138 & -0.055 \\
\hline
\end{tabular}

Unskewed data distribution. ${ }^{a}$ Adjusted for sex. ${ }^{b}$ Adjusted for sex and specific drug treatment. 